\title{
A Model of the Dynamic Error as a Measurement Result of Instruments Defining the Parameters of Moving Objects
}

\author{
D. Dichev ${ }^{1}$, H. Koev ${ }^{1}$, T. Bakalova ${ }^{2}$, P. Louda ${ }^{3}$ \\ ${ }^{1}$ Department of Machine and Precision Engineering, Faculty of Machine and Precision Engineering, \\ Technical University of Gabrovo, 4 Hadji Dimitar Street, 5300 Gabrovo, Bulgaria, \\ dichevd@abv.bg,koevh@abv.bg \\ ${ }^{2}$ Institute for Nanomaterials, Advanced Technologies and Innovation, Technical University of Liberec, \\ Studentska st. 2, 46117 Liberec, Czech Republic, tbakalova@seznam.cz \\ ${ }^{3}$ Department of Material Science, Technical University of Liberec, 2 Studentska Street, \\ 46117 Liberec, Czech Republic, petr.louda@tul.cz
}

\begin{abstract}
The present paper considers a new model for the formation of the dynamic error inertial component. It is very effective in the analysis and synthesis of measuring instruments positioned on moving objects and measuring their movement parameters. The block diagram developed within this paper is used as a basis for defining the mathematical model. The block diagram is based on the set-theoretic description of the measuring system, its input and output quantities and the process of dynamic error formation. The model reflects the specific nature of the formation of the dynamic error inertial component. In addition, the model submits to the logical interrelation and sequence of the physical processes that form it. The effectiveness, usefulness and advantages of the model proposed are rooted in the wide range of possibilities it provides in relation to the analysis and synthesis of those measuring instruments, the formulation of algorithms and optimization criteria, as well as the development of new intelligent measuring systems with improved accuracy characteristics in dynamic mode.
\end{abstract}

Keywords: dynamic error, measurement in dynamic mode, inertial effects, parameters of moving objects, set-theoretic model.

\section{INTRODUCTION}

$\mathrm{T}_{0}$ HE DISTINGUISHING FEATURE of the present stage of development of measuring equipment is the expansion of the area of application of instruments measuring physical quantities that change in time. This is linked to the emergence of new areas of application of measuring instruments and the complication of the metrological problems solved with their help. One of these areas is very topical today since it is related to the development and improvement of measuring equipment that determines the parameters characterizing the space-time position, the movement mode, etc. of ships, aircrafts and road vehicles. The steering effectiveness of these moving objects depends on the quality (accuracy, reliability, form and rate of presentation) of the measurement information.

The main characteristic of the quality of the measuring instruments is their accuracy. The distinguishing feature of the above mentioned measuring instruments is that they operate under conditions of dynamic influences determined by the movement of the moving objects or the fluctuations of the ships, aircrafts and road vehicles, as well as by the vibrations in the place where the measuring instruments are positioned.

The above listed movements of the moving objects cause inertial forces and moments. The latter establish conditions under which the inertial components of the measuring instruments positioned on the hull, fuselage or body of the moving objects operate in an inertial field of force. Under the influence of the latter the inertial components change their laws of movement [1]. As a result some energy is accumulated and it changes their forecast movement. The accumulated energy determines the own fluctuations of the inertial system. However, some external energy is also continuously transferred to the system. It defines the forced fluctuations. Consequently the overall movement of the instrument sensitive elements may considerably differ from the nominal one, thus leading to inaccuracy in the measurement result. This inaccuracy is quantitatively expressed by means of the dynamic error. Actually, this is one of the components of the dynamic error but within instruments measuring the parameters of moving objects this is the main component and it often obtains such values that it can be identified with the total dynamic error, i.e., the other components have a negligible effect on its formation. Due to its specific nature this component is often referred to as an inertial component [2]. Research in this area indicates [3], [4] that if there are not adequate solutions in the metrological procedures of the measuring instruments, the inertial component can obtain considerable values which lead to the uncertainty of the measurement result.

Therefore, the inertial component of the dynamic error is crucial for the formation of the accuracy parameters of this type of measuring instruments. From this perspective, the development of an accurate, efficient and logically consistent (in relation to metrological processes) model is an important condition for the solution of problems related to both the analysis and synthesis of the measuring instruments.

On the other hand, the dynamic error models presented in specialized literature [3-5] are not concrete enough and do not reflect the specific nature of the formation of the dynamic error inertial component considered in this paper. This circumstance reduces to a great extent the possibilities for investigating the characteristics of the dynamic error, as 
well as for the synthesis of the instruments in relation to the block diagram, characteristics and measurement procedure according to the dynamic error minimum criteria.

The abovementioned justifies the elaboration of the present paper whose aim is to develop a structural and mathematical model of the dynamic error with a view to their application in the analysis and synthesis of measuring instruments determining the parameters of moving objects.

\section{BASIC CONSIDERATIONS}

The metrological theory related to dynamic measurement aims to achieve traceability at the accuracy level required by today's science. [4]. In this respect the following main tasks emerge:

- transferring the value of the measuring unit from the reference to the operating instruments under the conditions of metrological traceability;

- standardizing and defining the dynamic characteristics of the measuring instruments;

- estimating and correcting the dynamic error.

As it can be seen, dynamic measurements can be structured in the common metrological concept of measurements but the content of and the methods for completing the well-known tasks are specific. For example, the analysis of the dynamic errors should be based on a number of starting principles which can be summarized as follows:

\section{A. Principal equation.}

The measurement error $\delta_{\Sigma}$, according to VIM, is the difference between the measurement result $Q$ and the referent value of the quantity under measurement $Q_{r v}$, i.e. [6]

$$
\delta_{\Sigma}(t)=Q(t)-Q_{r v}(t)
$$

In (1) the three quantities must be of equal dimension. Then error $\delta_{\Sigma}(t)$ will be a time variable quantity because the dynamics of the measured quantity $Q_{r v}(t)$ follows. When measuring the time variable quantity, the error is a determined or random function of time.

\section{B. Measurement mode.}

The three quantities in (1) are unified by the concept of dynamic measurement mode. From a mathematical viewpoint the formulation of the operating mode is the first step of developing the dynamic error models since the time component $t$ is introduced by it. The latter is an argument in the functions describing the input and output processes and the transfer and weighting functions. It defines the relationships required to express the basic concepts in the dynamic measurements.

In the present paper, by a dynamic measurement mode we mean such a mode where the instrument is able to measure the dynamics of the input quantity or there is at least one unit in the instrument which operates in this mode, and the dynamic error in the result can obtain values which cannot be neglected [7]. This formulation of the measurement mode is based on the widest possible set of characteristics building the measuring environment such as: the nature of the measured quantity, the type of the transfer function, the duration of the transfer period, the ability of the measuring instrument to measure in dynamic mode, the influence of the dynamic error on the formation of the measurement result.

\section{Investigation approach.}

When investigating the errors in dynamic measurement mode, according to the principal equation (1), it is expedient to use the approach applied in static measurements. It includes an analysis of the measurement errors, their division into separate components, theoretical and experimental investigation of each component, a synthesis of the total error usually on the basis of the sum of the estimates of the separate components. The main disadvantage of this approach is determined by the unavoidable loss of accuracy at the synthesis stage. On the other hand, the possibility for accurate estimation of the separate components on the basis of thorough investigation of their sources and the possibility for reducing their values by introducing adequate methods provide greater advantages in comparison with the disadvantage cited above.

\section{Specific nature of the dynamic error.}

The investigation of measurement errors is specific for each measurement task. In this respect the characteristics of the above described inertial component of the dynamic error have their specific nature. Therefore, the development of an accurate dynamic error model adequately reflecting the physical nature of its formation and possessing algorithmic logic in relation to the metrological determination of processes is an important precondition for the successful implementation of the analysis and synthesis of this type of measuring instruments.

\section{STRUCTURAL MODEL}

The present structural dynamic error model is based on the set-theoretic description of the measuring system and its input and output quantities (Fig.1.). This model differs from the dynamic error models described in specialized literature [3-5] since the structural solution is subject to the processes forming the inertial component.

In this sense the block diagram is structured in such a way that the non-inertial and inertial components building up the instrument are provisionally divided in separate modules.

\section{A. Measurement result.}

To obtain information on the state of the moving object by using the measuring instrument (MI), the current values of the components of the input coordinates vector $[\mathrm{X}]$ are measured (Fig.1.). As it is known, the measurement procedure cannot be implemented without errors. Therefore, the measurement result vector [Y] at the output of MI will be formed by

$$
[\mathbf{Y}]=[\mathbf{X}]+\left[\Delta_{\Sigma}\right],
$$

where $\left[\Delta_{\Sigma}\right]$ is the vector of the total error. 


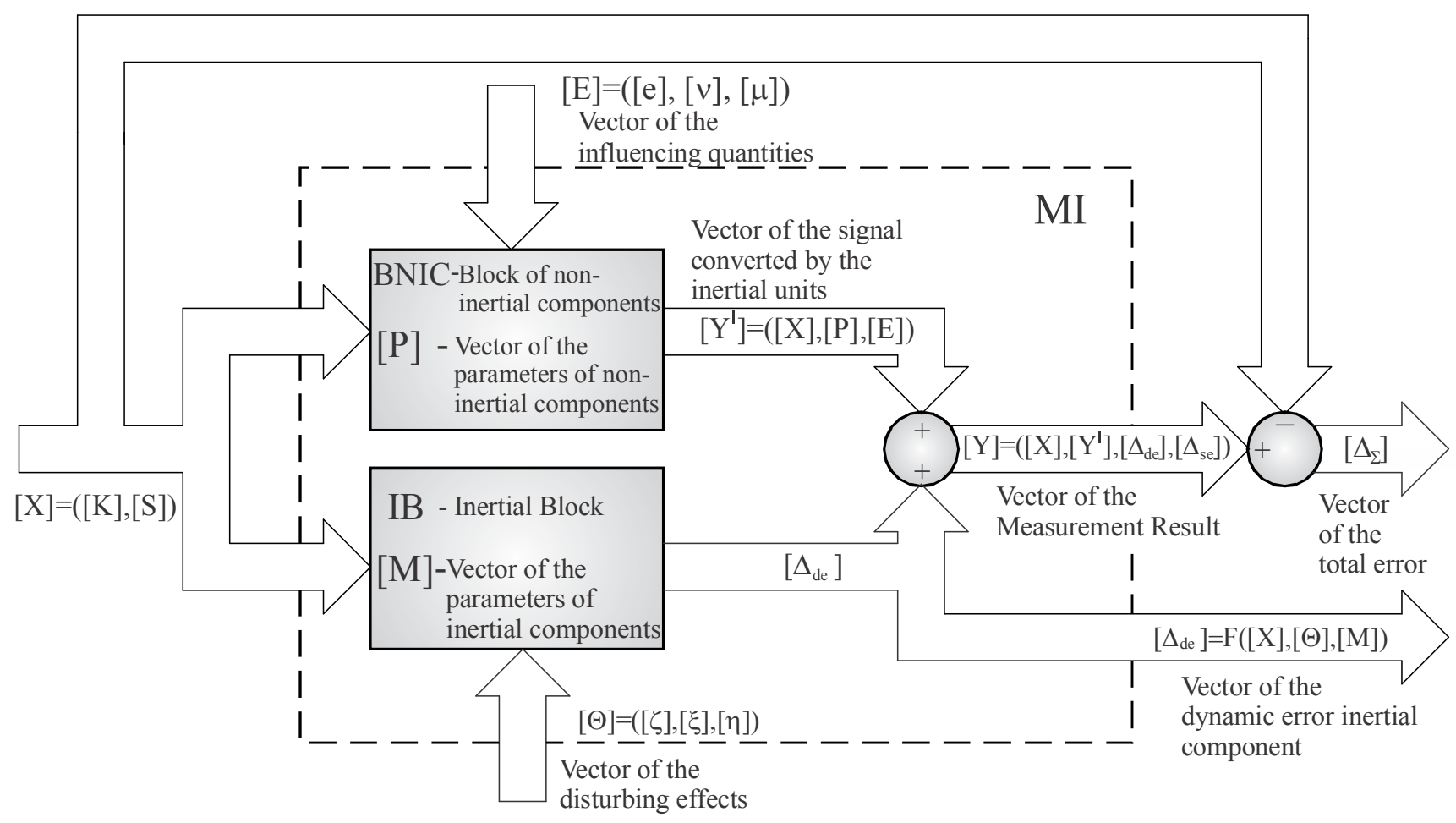

Fig.1. A set-theoretic model of the measuring system.

In terms of the dynamics of the processes forming the total error, its vector can be divided into two components - the vector of the static error $\left[\Delta_{\mathrm{se}}\right]$ and the vector of the dynamic error $\left[\Delta_{\mathrm{de}}\right]$.

Vector $\left[\Delta_{\text {se }}\right]$ is formed under the influence of the vector of the deviations of the non-inertial component parameters $[\Delta \mathbf{P}]$, the vector of the influencing quantities $[\mathbf{E}]$ and the vector of the measured quantity $[\mathbf{X}]$ if there is a multiplicative component. Then the vector of the signal converted by the non-inertial components $\left[\mathbf{Y}^{\prime}\right]$ will be determined by the functional

$$
\left[\mathbf{Y}^{\prime}\right]=\mathbf{F}\{[\mathbf{X}],[\mathbf{P}],[\mathrm{E}]\},
$$

where $[\mathbf{P}]$ is the vector of the parameters of the non-inertial components.

\section{B. Dynamic error.}

Actually, some of the components of the above listed vectors participating in the formation of vector $\left[\Delta_{\mathrm{se}}\right]$ determine the occurrence of particular dynamic error components. As it has already been mentioned, within the present study those components have negligible influence on the formation of the dynamic error. Therefore, they will not be discussed in this paper.

The vector of the dynamic error inertial component is defined by the functional of the following vector elements:

$$
\left[\Delta_{\mathrm{de}}\right]=\mathbf{F}_{\mathrm{de}}\{[\mathbf{X}],[\Theta],[\mathbf{M}]\},
$$

where $[\Theta]$ - a vector of the disturbing inertial effects; $[\mathbf{M}]-$ a vector of the parameters of the inertial components.
The model in Fig.1. is provisional in relation to the actual conversion processes in the measuring circuit but it accurately reflects the set-theoretic description which can be used as a base for building up the required mathematical models. The purpose of the model is to objectify the investigation into the influence of the dynamic processes on the measurement accuracy. In this way the set of inertial components can be viewed as a single block with respective input and output parameters, thus resulting in a wide range of possibilities for mathematical modeling of the determining processes.

\section{MATHEMATICAL MODEL OF THE DYNAMIC ERROR}

The most felicitous estimation of the dynamic error is done by means of its root-mean-square model. It is determined by the fact that in the presence of a random process $x(t)$ at the input of the measuring instrument, the dynamic error at its output

$$
\delta_{d e}(t)=y(t)-x(t)
$$

will also be a random function of time and the detailed information about it cannot be obtained by its instantaneous values. Thus, the mathematical model of the dynamic error should be based on some average values for which it is aptly to use the root-mean-square value [8-10], provided that process $x(t)$ possesses the quality of ergodicity, i.e.

$$
\delta_{d e}(t)=\sqrt{\overline{[y(t)-x(t)]^{2}}}=\sqrt{\lim _{T \rightarrow 0} \frac{1}{T} \cdot \int_{0}^{T}[y(t)-x(t)]^{2} \cdot d t} .
$$


The definition of $\delta_{d e}(t)$ as a separate component in the measuring system model makes it possible to include it in the overall logic of the input-output processes. In this way the required mathematical models defining the formation of $\delta_{d e}(t)$ in the context of the dynamic conditions of the problem, which are closest to the real-life processes, can be developed.

Therefore, the present paper presents a new concept of modeling and investigating the dynamic error. This concept is based on the mathematical model of function $\delta_{d e}(t)$ as a single component possessing certain characteristics and participating in the formation of the measurement result. Thus the approach chosen provides a possibility not only for investigating the characteristics of the dynamic error but also for developing algorithms, methods and tools [11] for its elimination as a single component.

On the ground of the above mentioned, new intelligent measuring systems could be developed, whose improved accuracy characteristics in dynamic measurement mode are formed on the basis of elimination of the dynamic error in real time [12]. That measuring method can replace the approaches used so far in the measuring instruments since they have a number of disadvantages such as: sophisticated structure, insufficient reliability in extreme conditions, they require special systems to ensure their operation, big size, high price, etc. [13]. Hence, the development and improvement of those measuring systems could be a perspective branch in the field of dynamic measurements.

The characteristics of function $\delta_{d e}(t)$ depend on the qualities of the input processes and the parameters of the measuring instrument inertial block. The dynamics of the linear inertial systems is determined by linear differential equations. In general, the relationship between the output coordinate defining the dynamic error and the input parameters is described by a system of linear differential equations or by an equation of the type

$$
\begin{aligned}
& F\left[\delta_{d e}(t), \dot{\delta}_{d e}(t), \ddot{\delta}_{d e}(t), \ldots, \delta_{d e}{ }^{(n)}(t) ; x, \dot{x}(t), \ddot{x}(t), \ldots, x^{(m)}(t),\right. \\
& \left.q(t), \dot{q}(t), \ddot{q}(t), \ldots, q^{(k)}(t) ; t\right]=0 .
\end{aligned}
$$

where $q(t)$ are inertial effects.

Upon measuring the dynamically changing quantity, an inverse problem is solved - a recovery of the input signal by the signal measured by the instrument. This problem is related to the elimination of the dynamic error from the measurement result. Upon modeling the dynamic error according to the set-theoretic description in Fig.1., the structure of the direct problem is used. In this case the sought solution (the dynamic error) is the output coordinate. The latter depends on the vector of the input coordinates $[X]$, the vector of the disturbing inertial effects $[\Theta]$ and the vector of the own parameters of the components of the inertial block $[\mathbf{M}]$.

The vector of the input coordinates $[\mathbf{X}]$ takes part in the formation of the dynamic error by means of its inertial characteristics that form along with vector $[\mathbf{M}]$ inertial forces and moments. The same is true for the vector of the disturbing inertial effects $[\Theta]$. However, this vector does not contain informative parameters for the measuring system.
An important condition for the solution of the problem related to the definition of the dynamic error mathematical model is that equation (7) should be written in such a way that makes it possible to define the coordinate expressing the deviation of the inertial components from their nominal current position depending on the measured quantity and the disturbing effects. If all the coefficients on the left and right side of the differential equation are constant, then the transfer function $W(p)$ of the inertial block in relation to the input quantity $x(t)$ can be presented in the form of a fractionrational function in relation to the operator $p$ [14]. If a stationary random signal $x(t)$ having known spectral density $S_{x}(\omega)$ or a known correlation function $K_{x}(\tau)$ enters at the input of the linear inertial block of the measuring instrument with a known transfer function $W(p)$, then the output signal characterizing, in this case, the dynamic error will also be a stationary signal [15]. The spectral density of the established output signal can be determined by

$$
S_{\delta_{d e}}=A(\omega)^{2} \cdot S_{x}(\omega)
$$

where $A(\omega)=|W(i \cdot \omega)|$ is the amplitude-frequency function of the inertial block.

Usually at the input of the inertial block not only the measured quantity enters but also the disturbing effects $q(t)$. In this case, when developing the mathematical model, it is necessary to consider all quantities operating at the input. In addition, if those quantities are independent and stationary, the principle of superposition (superposition of signals) is applied, i.e. [15]

$$
S_{\delta_{d e}}(\omega)=A(\omega)^{2} \cdot S_{x}(\omega)+A(\omega)^{2} \cdot S_{q}(\omega),
$$

where $S_{q}(\omega)$ - the spectral density of the disturbing quantity. Within known spectral density $S_{\delta_{d e}}$, the corresponding correlation function can be determined by [16]

$$
K_{\delta_{d e}}(\tau)=\frac{1}{\pi} \cdot \int_{0}^{\infty} S_{\delta_{d e}}(\omega) \cdot \cos (\omega \cdot \tau) \cdot d \tau .
$$

Then the dispersion of the dynamic error will be:

$$
D_{\delta_{d e}}=K_{\delta_{d e}}(0) \text { or } D_{\delta_{d e}}=\frac{1}{\pi} \cdot \int_{0}^{\infty} S_{\delta_{d e}}(\omega) \cdot d \omega
$$

Since the dispersion is a root-mean-square form of representation of the dynamic error values, the root-meansquare estimate of the latter can be obtained by

$$
\sigma_{\delta_{\partial e}}=\sqrt{D_{\delta_{d e}}^{2}}
$$

Actually, the estimate (12) coincides by meaning with the root-mean-square model (6) of the dynamic error but it is developed according to the characteristics of the block diagram in Fig.1. It is a good reason to reduce the final mathematical model of the dynamic error to the following equation: 


$$
\delta_{d e}=\sigma_{\delta_{d e}} .
$$

The maximal values the dynamic error can reach can be determined by (13). These values are calculated with the help of the formula

$$
\delta_{d e}^{\max }=H \cdot \sigma_{\delta_{d e}},
$$

where $H$ is a coefficient that depends on the law according to which the ordinates of the process $\delta_{d e}(t)$ defining the dynamic error are distributed.

The estimates (12) and (13) can be complemented with the mathematical expectation $M\left[\delta_{d e}\right]$ of the process $\delta_{d e}(t)$, which for stationary random time functions is a constant quantity [16] and provides significant information on the characteristics of the dynamic error.

To visualize the logic of functioning of the proposed model, the process of formation of the dynamic error for a specific measuring instrument will be considered. The latter is a heelmeter measuring the heel and the roll of a ship. These measuring instruments possess sensitive elements of inertial qualities which model the local vertical. In this way they set the supporting coordinate system in the metrological circuit of the instrument. The current values of the angles determining the heel and the roll of the ship are measured according to the supporting system set. Therefore, each deviation of the sensitive element from the actual position of the local vertical results in a measurement error. The deviations of the sensitive elements are caused by the inertial effects determined by the dynamic characteristics of the measured quantity and the disturbing effects. Expressed in a time function, the deviations are defined as stationary random process, and expressed in a mathematical form, depending on their degrees of freedom, they are obtained as solutions to one or more equations of the type (7).

When the sensitive element of the heelmeter is a module of a fluctuation unit type, its transfer function is expressed by

$$
W(p)=\frac{1}{T^{2} \cdot p^{2}+2 \cdot \xi \cdot T \cdot p+1} .
$$

where $T$ - the time constant of the instrument; $\xi$ - a relative damping factor.

In the most reduced case the right side of the differential equation describing the deviations of the sensitive elements according to the measuring coordinate is formed by the function

$$
X(t)=z \cdot \ddot{\theta}(t),
$$

where $z$ - the coordinate in the vertical direction of the suspension point of the sensitive element with regard to the center of gravity of the ship; $\ddot{\theta}(t)$ - the second derivative of the process defining the roll of the ship, which in practice is the measured quantity.

Then the spectral density of (16) will be

$$
S_{x}(\omega)=z^{2} \cdot S_{\ddot{\theta}}(\omega)=z^{2} \cdot \omega^{4} \cdot S_{\theta}(\omega) .
$$

On the other hand, the spectral density $S_{\theta}(\omega)$ of the roll process can be approximated accurately enough by

$$
S_{\theta}(\omega)=\frac{2 . D_{\theta} \cdot \mu_{\theta}}{\pi} \cdot \frac{b_{\theta}^{2}}{\omega^{4}+2 \cdot a_{\theta}^{2} \cdot \omega^{2}+b_{\theta}^{4}},
$$

where $D_{\theta}$ - the dispersion of the roll fluctuations; $b_{\theta}^{2}=\mu_{\theta}^{2}+\lambda_{\theta}^{2} ; a_{\theta}^{2}=\mu_{\theta}^{2}-\lambda_{\theta}^{2} ; \mu_{\theta}$ - the damping factor of the correlation function of the process $\theta(t) ; \lambda_{\theta}$ - the roll frequency in calm water.

According to (8) the spectral density of the dynamic error will be:

$$
\begin{aligned}
& S_{\delta_{d e}}(\omega)=\frac{1}{\left(1-T^{2} \cdot \omega^{2}\right)^{2}+4 \cdot \xi \cdot T^{2} \cdot \omega^{2}} \times \\
& \times \frac{2 \cdot D_{\theta} \cdot \mu_{\theta} \cdot z^{2}}{\pi} \cdot \frac{b_{\theta}^{2} \cdot \omega^{4}}{\omega^{4}+2 \cdot a_{\theta}^{2} \cdot \omega^{2}+b_{\theta}^{4}} .
\end{aligned}
$$

After using (11) and (19), the following is obtained

$$
\begin{aligned}
& D_{\delta_{d e}}=\frac{1}{\pi} \cdot \int_{0}^{\infty} S_{\delta_{d e}}(\omega) \cdot d \omega= \\
& \frac{1}{\pi} \cdot \int_{0}^{\infty} \frac{1}{\left(1-T^{2} \cdot \omega^{2}\right)^{2}+4 \cdot \xi \cdot T^{2} \cdot \omega^{2}} \times \\
& \times \frac{2 \cdot D_{\theta} \cdot \mu_{\theta} \cdot z^{2}}{\pi} \cdot \frac{b_{\theta}^{2} \cdot \omega^{4}}{\omega^{4}+2 \cdot a_{\theta}^{2} \cdot \omega^{2}+b_{\theta}^{4}} \cdot d \omega .
\end{aligned}
$$

Since the components in (19) - a transfer function and spectral density, are fraction-rational functions of $\omega$, the dispersion $D_{\delta_{d e}}$ can be calculated by analytical methods without defining the roots of the denominator and decomposing the subintegral expression into simple fractions. The definition of $D_{\delta_{d e}}$ is reduced to the calculation of a table integral of the type [16]

$$
J_{n}=\frac{1}{\pi} \cdot \int_{0}^{\infty} \frac{M(j \cdot \omega)}{|H(j \cdot \omega)|^{2}} \cdot d \omega
$$

where $M(j . \omega)$ and $H(j . \omega)$ are polynomials of the following structure:

$$
\begin{aligned}
& M(j \cdot \omega)=b_{0}(j \cdot \omega)^{2 \cdot(n-1)}+b_{l}(j \cdot \omega)^{2 \cdot(n-2)}+\ldots+b_{n-1} ; \\
& H(j \cdot \omega)=a_{0}(j \cdot \omega)^{n}+a_{I}(j \cdot \omega)^{n-1}+\ldots+a_{n} .
\end{aligned}
$$

In the above expressions $a_{0}, \ldots, a_{n}, b_{0}, \ldots, b_{n-1}$ are constant coefficients depending on the parameters of the sensitive element. Polynomial $M(j . \omega)$ contains only the even powers of $(j . \omega)$ and polynomial $H(j . \omega)$ - all powers. 
Then on the basis of (20), the solution of (19) is obtained in the type

$$
D_{\delta_{d e}}=\frac{D_{\theta} \cdot b_{\theta}^{2} \cdot z^{2}}{g^{2}} \cdot \frac{b_{\theta}^{2}+\frac{\mu_{\theta}}{\xi \cdot T}}{Q^{2}+4 \cdot R},
$$

where the following expressions are denoted with $Q$ and $R$

$$
\begin{gathered}
Q=1-T^{2} \cdot b_{\theta}^{2} ; \\
R=\left(T^{2} \cdot b_{\theta}^{2} \cdot \xi+T \cdot \mu_{\theta}\right) \cdot\left(\xi+T \cdot \mu_{\theta}\right) .
\end{gathered}
$$

Expression (22) is a good example of the usefulness of the proposed dynamic error model. The equations worked out on the basis of this model explicitly express the relationship between the dynamic error and the parameters influencing its formation. Therefore it is possible to not only analyze the influence of those parameters on the dynamic error characteristics but also to develop methods for the parametric synthesis of the measuring instrument in relation to its dynamic accuracy.

The influence of the basic parameters on the size of the dynamic error is illustrated in Fig.2. and Fig.3. It can be seen that the parameters of the inertial components affect to some extent the size of the dynamic error. However, this influence is not so considerable, so that the synthesis of similar measuring instruments can be based only on the optimal selection of the values of those parameters. Therefore, the synthesis of this type of measuring instruments in relation to the dynamic accuracy should be based on other methods. One of these approaches is to include additional measurement channels, operating in parallel with the main channels, in the metrological circuits. The additional channels could have the specific structure of correcting devices intended to eliminate the dynamic error.

\section{CONCLUSION}

The proposed dynamic error model developed on the basis of a new block diagram has a number of advantages related to the analysis and synthesis of measuring instruments positioned on moving objects and measuring the basic parameters. The effectiveness of the model is due to a new concept based on the dynamic error function presented as a single component and obtained as a result of the logical sequence of the physical processes that form it. This approach makes possible the definition of formulae specifying the relationship between the dynamic error and its determining parameters, which is an important condition for the successful solution of the problems emerging at the design stage of the measuring instruments viewed in the present paper.

The mathematical model of the dynamic error is developed according to its root-mean-square estimate, which is of great importance in terms of application since it expands the possibilities for formulating optimization algorithms and criteria.
The definition of the dynamic error as a specific process determined by the input quantities and parameters of the measuring instrument provides the required mathematical tools in the area of measuring engineering for developing new intelligent measuring systems whose improved accuracy characteristics in dynamic measurement mode are formed on the basis of the dynamic error elimination in real time.

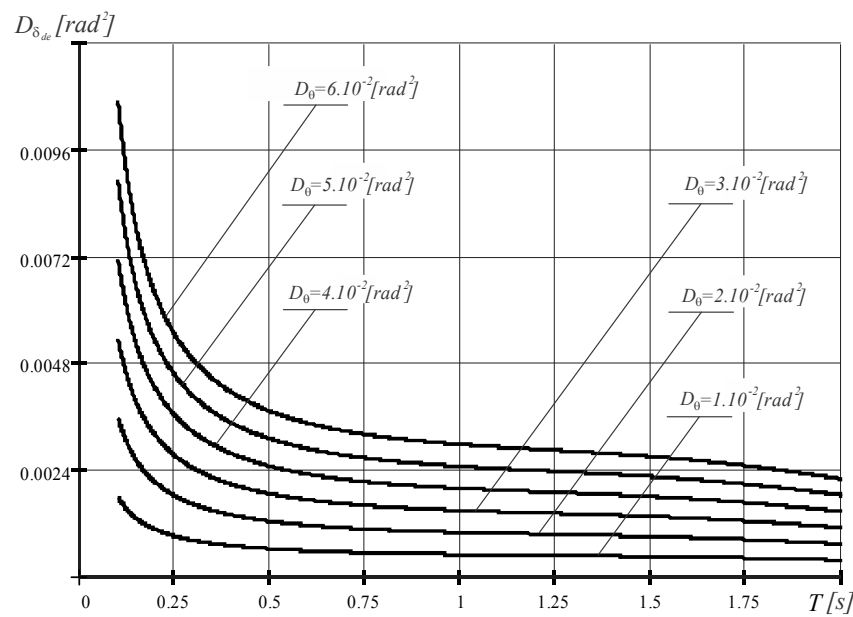

Fig.2. Graphic dependences between the dynamic error dispersion and the parameters influencing its formation in a function of the time constant $T$ for different values of the dispersion $D_{\theta}$.

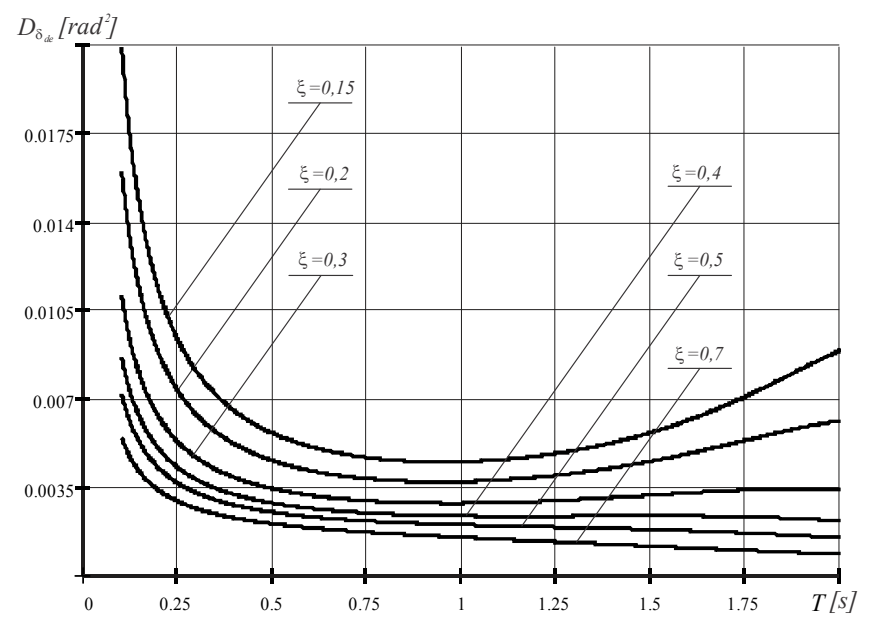

Fig.3. Graphic dependences between the dynamic error dispersion and the parameters influencing its formation for different values of the damping factor $\xi$.

\section{ACKNOWLEDGMENT}

The research reported in this paper was supported in part by the Project Stimulation of Scientific Research within the Public Higher Institutions, Scientific Research Fund Bulgaria DDVU02/15/20.12.2010, as well as by the Project OP VaVpI Centre for Nanomaterials, Advanced Technologies and Innovation CZ.1.05/2.1.00/01.0005 and by the Project Development of Research Teams of R\&D Projects at the Technical University of Liberec CZ.1.07/2.3.00/30.0024. 


\section{REFERENCES}

[1] Dichev, D.A., Golemanova, S.H. (2010). Characteristics of the results upon measuring signals representing random processes. In Metrology and Metrology Assurance : XX National Scientific Symposium, 10-14 September 2010, Sazopol, Bulgaria, 51-57.

[2] Dichev, D.A., Koev, H.C. (2012). Increase of dynamic accurasy in measurement systems of parameters of moving objects. In Metrology and Metrology Assurance : XXII National Scientific Symposium, 1115 September 2012, Sazopol, Bulgaria, 57-64.

[3] Nazarov, N.G. (2002). Metrology. Basic Concepts and Mathematical Models. Moscow : Vishshaya Shkola.

[4] Pronkin, N.S. (2003). Basics of Metrology for Dynamic Measurements. Moscow : Logos.

[5] Granovskij, V.A. (1984). Dynamic Measurements. St. Petersburg : Energoatomizdat.

[6] Joint Committee for Guides in Metrology. (2008). International vocabulary of metrology - Basic and general concepts and associated terms (VIM). JCGM 200:2008.

[7] Dichev, D.A. (2008). Mathematical models of parameters defining a mode of measurement. In Metrology and Metrology Assurance : XVIII National Scientific Symposium, 9-12 September 2008, Sazopol, Bulgaria, 86-92.

[8] Ventcel, E.S., Ovcharov, L.A. (2007). Random Processes Theory and Its Engineering Application. Moscow : Vishaya Shkola.
[9] Kamenský, M., Kovac, K. (2011). Correction of ADC errors by additive iterative method with dithering. Measurement Science Review, 11 (1), 15-18.

[10] Hegeduš, H., Mostarac, P., Malarić, R. (2011). Comparison of RMS value measurement algorithms of non-coherent sampled signals. Measurement Science Review, 11 (3), 79-84.

[11] Venkatesh, A.K., Mathivanan, N. (2012). Design of MEMS accelerometer based acceleration measurement system for automobiles. Measurement Science Review, 12 (5), 189-194.

[12] Dichev, D., Koev, H., Bakalova, T., Louda, P. (2013). An algorithm for improving the dynamic accuracy in systems for measuring the parameters of moving objects. International Journal of Engineering, Business and Enterprise Applications, 5 (2), 1-9.

[13] Sveshnikov, A.A., Rivkin, S.S. (1974). Probability Methods in the Applied Gyroscope Theory. Moscow : Nauka.

[14] Sveshnikov, A.A. (2011). Applied Methods from the Random Functions Theory. Moscow : Lan.

[15] Baskakov, S.I. (2000). Radio Engineering Chains and Signals. Moscow : Vishaya Shkola.

[16] Stepetov, A.G. (2006). Theory, Calculation and Design of Measuring Instruments. Moscow : Standartinform.

Received November 19, 2013. Accepted July 31, 2014. 